Original Article

\title{
The effects of new taping methods designed to increase muscle strength
}

\author{
Tsutomu Fukui, RPT, PhD ${ }^{1)^{*}}$, Yuko Otake, RPT, PhD ${ }^{1)}$, Takashi Kondo, RPT, MS ${ }^{1)}$ \\ 1) Health Care Science, Graduate School, Bunkyo Gakuin University: 1-19-1 Mukogaoka, Bunkyo-ku,
} Tokyo 113-8668, Japan

\begin{abstract}
Purpose] Although there are several studies on the use of elastic tape to influence muscle strength, results are contradictory and controversial. Our previous studies based on the sliding mechanism between superficial fascia and subcutaneous tissue may help the muscle strength. The purpose of this study was to confirm the effects of new taping methods on muscle strength. [Subjects and Methods] Sixteen healthy male participants took part in this study. Tape was applied on the right gluteus maximus and hip extension strength was determined by an isokinetic evaluation $(30 \% \mathrm{sec}$, concentric mode, four conditions). Condition 1: Tape was applied from the muscle insertion to origin; Condition 2: Tape was applied from the origin to insertion; Condition 3: Dummy tape with no direction; Condition 4: No tape was applied. [Results] The mean value of conditions 1-4 were 398.2 \pm 24.3 Newton (N), 343.7 $\pm 25.9 \mathrm{~N}, 363.7 \pm 26.4 \mathrm{~N}$, and $371.3 \pm 26.3 \mathrm{~N}$, respectively (mean $\pm \mathrm{SE}$ ). The result of condition 1 was significantly greater compared with the other conditions. [Conclusion] This new method corresponded to a tape direction of insertion-rigin may help to increase the muscle strength.

Key words: Muscle strength, Taping, Skin
\end{abstract}

(This article was submitted Aug. 30, 2016, and was accepted Sep. 29, 2016)

\section{INTRODUCTION}

The techniques of elastic taping are used for various objectives in sports or related therapeutic approaches. Kinesio taping ${ }^{\circledR}[\mathrm{KT}]$, one of the elastic taping techniques available today, is used to reduce pain, improve range of motion, influence muscle strength, proprioception, and muscle activity, and reduce lymphedema and peripheral venous flow ${ }^{1,2)}$. Although there are several studies that have described the use of elastic tape to influence muscle strength, their results are contradictory and controversial.

For instance, maximum grip strength was measured under three conditions: [1] without taping, [2] with placebo taping, and [3] with KT. KT was applied on the wrist flexor of the participants in the healthy group ${ }^{3}$ and of athletes with medial elbow epicondylar tendinopathy ${ }^{4}$. The results of the two groups showed no significant difference among the three conditions. Furthermore, it was evidenced that by applying KT on the wrist extensors the maximum grip strength did not change ${ }^{5)}$. In contrast, another article reported the effects of KT on handgrip strength ${ }^{6)}$. The participants were divided into three groups: KT, KT without tension, and no tape. The Kinesio group showed significant increase in handgrip strength after taping. When analyzing these reports, positive and negative results on the effect of elastic tape on muscle strength are evidenced.

Another area of study refers to femoral quadriceps strength. Studies present contradictory results. A research study where KT was applied on the quadriceps ${ }^{7)}$ showed that there was no significant change in knee extensor peak torque in healthy women with or without the use of tape. Another study assessed quadriceps strength under three conditions: [1] without taping, [2] immediately after taping, and [3] Twelve hours after taping, with the tape remaining in situ when taping was applied. The result showed no significant difference in muscle power among the three conditions ${ }^{8)}$. Additionally, studies have shown that the application of KT on the vastus medialis did not change muscle torque ${ }^{9)}$ but did change muscle tone ${ }^{10)}$. The latter

*Corresponding author. Tsutomu Fukui (E-mail: fukui@bgu.ac.jp)

(C2017 The Society of Physical Therapy Science. Published by IPEC Inc.

This is an open-access article distributed under the terms of the Creative Commons Attribution Non-Commercial No Derivatives (by-nc-nd) License $<$ http://creativecommons.org/licenses/by-nc-nd/4.0/>. 
study concluded that there was a clinically significant increase in the bioelectrical activity of the vastus medialis.

Another area of study was the effect of KT on stroke patients. In one such study, patients were divided into two groups: $\mathrm{KT}$ group and non-KT group ${ }^{11)}$. The results for knee extension and flexion on the paretic side showed that the changes in peak torque levels were significantly higher in the KT group compared with the control group. Moreover, the KT effect was evaluated on patients with osteoarthritis. The results proved that applying KT on the quadriceps improved knee peak torque ${ }^{12)}$.

Several researchers demonstrated KT's effectiveness on the gastrocnemius muscle. The influence of KT on the gastrocnemius, hamstrings, rectus femoris, and iliopsoas was tested by analyzing the jump performance of healthy elite track and field athletes ${ }^{13)}$. The results showed that KT has no effect on jumping performance. Similarly, it was tested whether KT of the gastrocnemius would influence muscle performance ${ }^{14}$. Results showed no significant difference in the outcomes between KT and placebo taping. Nevertheless, significant peak force increase was evidenced in the gastrocnemius immediately after $\mathrm{KT}^{15}$. In the first and second research studies ${ }^{13}{ }^{14)}$, the direction of the tape was from the muscle origin to its insertion point. On the contrary, tape direction in the last study was from the muscle's insertion point to its origin ${ }^{15}$.

To determine the overall tendency of the effect of KT application, three important systematic-review articles were studied. The first one employed a meta-analysis to study the effect of KT application from the standpoint of muscle strength ${ }^{2}$. The author concluded that there were some positive effects on the muscle's strength, although there were numerous trivial findings. The second systematic-review investigated four online databases. The data showed there was no evidence of muscle strength increase when applying $\mathrm{KT}^{16)}$. The third review investigated whether $\mathrm{KT}$ influences the muscle strength or not by employing a meta-analysis ${ }^{17}$. It revealed that the potential to increase muscle strength by using KT was negligible.

In spite of all these articles and their results, the question of why some researchers reported positive effects on muscle strength after using KT or elastic tapes remains. It was hypothesized that one of the differences between the positive and negative results might depend on the tape direction, tape and skin tension, and tape region.

Our initial focus was on the features of skin movement in clinical settings. These were studied by employing a threedimensional motion analysis system ${ }^{18)}$. The results of this study suggested that the skin moves according to certain physiological rules. Moreover, our recent study identified some of the physiological rules of the direction of skin movement during joint movement ${ }^{19)}$. The results showed that during pelvic anterior sway while standing, the skin on the front of the thigh would move upward while the back skin would move downward. Opposite skin movements were observed during posterior pelvic sway. It was also found that the lateral skin of the thigh moved upward and that the medial skin moved downward during hip adduction, and vice-versa during hip abduction. These findings indicate that skin movement based on the sliding mechanism between superficial fascia and subcutaneous tissue may influence the effects of elastic tape. To facilitate the increase of muscle strength, it was hypothesized that the tape and the skin should be stretched in the direction of insertionorigin while covering the related muscles.

The results of previous studies helped to develop new taping methods based on the physiological skin movements ${ }^{19)}$. The purpose of this study was to confirm the effects of these new taping methods on muscle strength, using an isokinetic torque machine.

\section{SUBJECTS AND METHODS}

This study was conducted as a randomized, cross-over design with a single group. KT was applied on the right gluteus maximus (GM), and hip extension strength was determined by using a Primus RS measurement machine (BTE technologies, Baltimore, USA).

Sixteen healthy male participants with a mean age of $22 \pm 2.1$ years, mean height of $172.0 \pm 2.84 \mathrm{~cm}$, and mean weight of $60.2 \pm 7.80 \mathrm{~kg}$ took part in this study. The participants were recruited from among the students of Bunkyo Gakuin University. All the participants understood the details of the study procedure and signed an informed consent prior to their participation in the study. Exclusion criteria included any history of lower limb musculoskeletal injury in the three months before the experiment. This study was approved by the Ethics Committee of Bunkyo Gakuin University (approval number, 2014MSJ03).

Following an adaptation period to the measurement machine, an isokinetic evaluation $(30 \% \mathrm{sec}$, concentric mode, four conditions) was conducted. The four conditions were as follows: condition 1: elastic tape was applied from the muscle point of insertion to its origin; condition 2: elastic tape was applied from the muscle's origin to its insertion point; condition 3: dummy tape with no particular direction was applied; condition 4: no tape was applied.

Taping methods were shown below. In condition 1, participants were asked to stand, flex their hip, and lie on their trunk on a bed. Since this position involved flexing the hip and extending the knee, the GM was stretched. Before applying the tape, the distance from the iliac crest to the gluteal sulcus was measured. Then, the tape was applied on the GM from its insertion point to its origin by using $20 \%$ stretch tension. Additionally, two short tapes $(5 \mathrm{~cm})$ were used; one was applied on the lower back of the trunk in the direction of the iliac crest, and another was placed on the thigh away from the gluteal sulcus (Fig. 1). These two short tapes exhibited the area of facilitation or inhibition of the skin area in opposite direction against main tape.

In condition 2, participants were in a prone position with the lower leg protruding off the bed. Tape was applied on the GM from its origin to its insertion point using $20 \%$ stretch tension. Two short tapes $(5 \mathrm{~cm})$ were applied; one was proximally on the lower back of the trunk away from the iliac crest, and another was on the back of the thigh in the direction of the gluteal sulcus. In condition 3, in the same position as condition 2, tape was placed on the GM with no tension. All tapes were $5 \mathrm{~cm}$ 


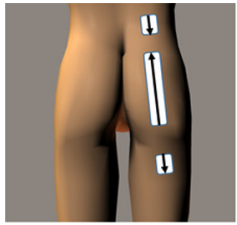

Condition 1

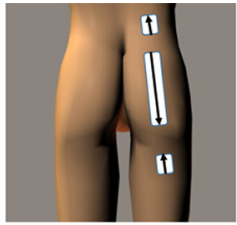

Condition 2

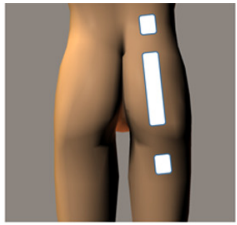

Condition 3

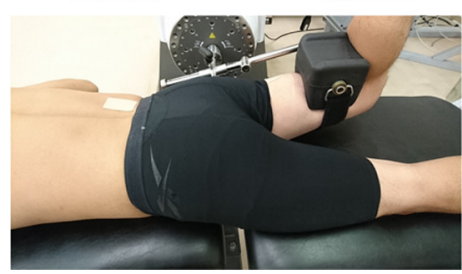

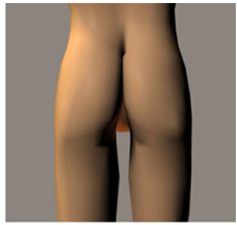

Condition 4

Condition 1: From insertion to origin

Participants were asked to stand, flex their hip, and lie on their trunk on a bed. Since this position involved flexing the hip and extending the knee, the GM was stretched. The tape was applied on the GM from its insertion point to its origin by using $20 \%$ stretch tension. Additionally, two short tapes $(5 \mathrm{~cm})$ were used; one was applied on the lower back of the trunk in the direction of the iliac crest, and another was placed on the thigh away from the gluteal sulcus

Condition 2: From origin to insertion

Participants were in a prone position with the lower leg protruding off the bed. Tape was applied on the GM from its origin to its insertion point using $20 \%$ stretch tension. Two short tapes $(5 \mathrm{~cm})$ were applied; one was proximally on the lower back of the trunk away from the iliac crest, and another was on the back of the thigh in the direction of the gluteal sulcus.

Condition 3: No direction

In the same position as condition 2, tape was placed on the GM with no tension.

Condition 4: No tape

in width (Multipore 2723-50, 3 M Japan, Tokyo, Japan).

Participants were asked to contract their GM to its maximum isokinetically. The measurement sequence of the four conditions was randomized, with an inter-condition duration of over five minutes. The differences in the mean value were assessed by using a one-way repeated measures ANOVA procedure. Analyses were conducted using SPSS 21.0 (IBM Japan, Tokyo, Japan). $\mathrm{P}$ values $<0.05$ were considered statistically significant.

\section{RESULTS}

The mean value of conditions $1-4$ were $398.2 \pm 24.3$ Newton $(\mathrm{N}), 343.7 \pm 25.9 \mathrm{~N}, 363.7 \pm 26.4 \mathrm{~N}$, and $371.3 \pm 26.3 \mathrm{~N}$, respectively (mean $\pm \mathrm{SE}$ ). A significant difference among the four conditions was observed. The mean value of condition 1 corresponded to a tape direction of insertion-origin, stretched skin condition, and final tape length $=120 \%$ of the original length; the result was significantly greater compared with the other conditions $(p<0.01)$. The mean value of condition 2 corresponded to a tape direction of origin-insertion, relaxed skin condition, and final tape length $=120 \%$ of the original tape length; the result was significantly lower compared with the other conditions $(p<0.05)$. No significant difference was observed between condition 3, dummy tape without direction, and condition 4, no tape. The results of maximal GM torque in each condition are presented in Tables 1, 2 .

\section{DISCUSSION}

When analyzing all four conditions, the results showed that only condition 1 facilitated better muscle strength. As previously described in the Introduction section, it was believed that tape direction was one of the most important factors in facilitating muscle strength. In Schiffer and Nunes' studies investigating the gastrocnemius, the common direction of taping was from origin to insertion. Their results showed no improvement in muscle strength ${ }^{13,14)}$. On the contrary, Lumbroso's study (tape direction=insertion-origin) showed positive effects on the gastrocnemius muscle strength ${ }^{15}$. In his study, the effect on hamstrings (tape direction=origin-insertion) was evaluated by using a hydraulic push dynamometer; the results showed no improvement in muscle strength. All these previous studies showed that the direction of tape was one of the most important factors in influencing muscle strength.

Several studies have analyzed the tension of the tape and skin on the quadriceps. The direction of the tape was origininsertion in all the articles ${ }^{7-9,11,12,20)}$. The tension between the tape and the skin varies with the study. Fu showed that the first and the last tape, which stand for both ends, were not stretched ${ }^{8}$. The first tape was placed inferior to the anterior superior 
Table 1. Hip extension strength

\begin{tabular}{lcccc}
\hline & Condition 1 & Condition 2 & Condition 3 & Condition 4 \\
\hline Hip extension (Newton) & $398.2 \pm 24.3$ & $343.7 \pm 25.9$ & $363.7 \pm 26.4$ & $371.3 \pm 26.3$ \\
\hline Mean \pm SE & & & \\
Condition 1: Main taping from insertion to origin of gluteus maximus & & \\
Condition 2: Main taping from origin to insertion of gluteus maximus & & \\
Condition 3: Main taping with no direction & & & \\
Condition 4: No tape & & &
\end{tabular}

Table 2. Comparison of each condition

\begin{tabular}{llcc}
\hline & & Mean difference & Standard error \\
\hline Condition 1 vs. & Condition 2 & $54.5^{* *}$ & 8.8 \\
& Condition 3 & $34.5^{* *}$ & 7.3 \\
& Condition 4 & $26.9^{* *}$ & 6.2 \\
Condition 2 vs. & Condition 1 & $-54.5^{* *}$ & 8.8 \\
& Condition 3 & $-20.0^{*}$ & 8.9 \\
& Condition 4 & $-27.6^{*}$ & 11.9 \\
Condition 3 vs. & Condition 1 & $-34.5^{* *}$ & 7.3 \\
& Condition 2 & $-20.0^{*}$ & 8.9 \\
& Condition 4 & -7.6 & 7.1 \\
Condition 4 vs. & Condition 1 & $-26.9^{* *}$ & 6.2 \\
& Condition 2 & $27.6^{*}$ & 11.9 \\
& Condition 3 & 7.6 & 7.1 \\
\hline$* * \mathrm{p}<0.01, * \mathrm{p}<0.05$ & & & Newton
\end{tabular}

iliac spine, and the last tape circled around the patella, ending at its inferior side. The portion between the first and the last tape was stretched to $120 \%$ length tension. Participants lay in a supine position with the hip flexed at $30^{\circ}$ and the knee flexed at $60^{\circ}$. Ekiz applied the rectus femoris tape on the same portion but without tension ${ }^{11)}$. Then the tape was crossed from the edges of the patella with maximum tension. Anandkumar described the tape as pulled with a $50-75 \%$ tension along the course of the rectus femoris until it reached the superior border of the patella ${ }^{12)}$. Lins applied the tape with $50 \%$ tension on the strip while the participants stood with the hip flexed at $0^{\circ}$ and the knee flexed at $90^{\circ}$ ). Wong described the tape tension as $75 \%$ of its maximal length tension ${ }^{9}$. In this way, each experimental method was different concerning the tension of both tape and skin. In these articles, one single principle could not be identified, even if Ekiz's and Anandkumar's papers evidenced a positive effect on the quadriceps strength ${ }^{11}{ }^{12}$. Therefore, these studies cannot be compared, since the conditions are not equal. This study presumed that tape and skin should be under tension to influence muscle strength.

Some researchers studied the influence on the grip strength of the region where the tape was applied. Chang determined the immediate effects of KT on healthy participants under three conditions: without taping, with placebo taping, and with $\mathrm{KT}^{3}$. Taping was applied from the wrist joint line to the medial epicondyle of the humerus. No significant results were found when analyzing these three conditions.

Nevertheless, Donec evaluated whether KT could increase the maximal grip strength ${ }^{21)}$. KT were used on the flexor digitorum superficialis, flexor digitorum profundus, wrist extensors, adductor policis, flexor policis brevis, and the first interosseous dorsalis. The results showed that the KT technique increased the maximal grip strength.

These results indicate that the applied region was important when trying to improve muscle strength. Furthermore, the region of tape was one of the most important factors when studying muscle strength.

From this study's results, it was concluded that guiding the skin in the direction of the muscle contraction could increase muscle strength. When the muscle moved from the insertion point to its origin point, guiding the skin in advance facilitated the contraction of the muscle. By using ultrasonic imaging, it was possible to observe the decrease of the resistance of the superficial fascia ${ }^{18)}$. When applying this method, it was necessary to move the skin toward the origin of the muscle to facilitate sliding of the skin. This study used the following method: the main taping direction was insertion-origin. Furthermore, two additional short tapes $(5 \mathrm{~cm})$ were used; one was applied proximally on the lower back in the direction of the iliac crest, while the other was placed on the thigh away from the gluteal sulcus. By using a $120 \%$ stretched tape, the skin was allowed to stretch. By doing all these, the muscle was able to perform better. In the case of the inhibitory method, all the tapes should be placed in a reverse direction, employing a 120\% stretched tape, and the skin should be relaxed. When guiding the skin in advance from origin-insertion, muscle contraction was blocked, thus reducing its muscle strength.

Previous studies have not clearly reported whether it was possible to increase muscle strength by using elastic tape and 
analyzing tape direction, tape tension, skin tension, and tape region. The advantage of this study contained all the factors mentioned above. This method may help physiological skin movement and muscle strength control.

This study has limitations, as it only investigated the immediate change of the muscle strength after the tape was applied. Moreover, only one method was used with respect to tape and skin tension.

\section{Conflict of interest}

None declared.

\section{REFERENCES}

1) Kase K, Wallis J, Kase T: Clinical therapeutic applications of the kinesio taping methods. Tokyo: Kinesio Taping Association, 2003.

2) Williams S, Whatman C, Hume PA, et al.: Kinesio taping in treatment and prevention of sports injuries: a meta-analysis of the evidence for its effectiveness. Sports Med, 2012, 42: 153-164. [Medline] [CrossRef]

3) Chang HY, Chou KY, Lin JJ, et al.: Immediate effect of forearm Kinesio taping on maximal grip strength and force sense in healthy collegiate athletes. Phys Ther Sport, 2010, 11: 122-127. [Medline] [CrossRef]

4) Chang HY, Cheng SC, Lin CC, et al.: The effectiveness of kinesio taping for athletes with medial elbow epicondylar tendinopathy. Int J Sports Med, 2013, 34: 1003-1006. [Medline] [CrossRef]

5) Cai C, Au IP, An W, et al.: Facilitatory and inhibitory effects of Kinesio tape: fact or fad? J Sci Med Sport, 2016, 19: 109-112. [Medline] [CrossRef]

6) Lemos TV, Pereira KC, Protássio CC, et al.: The effect of Kinesio Taping on handgrip strength. J Phys Ther Sci, 2015, 27: 567-570. [Medline] [CrossRef]

7) Lins CA, Neto FL, Amorim AB, et al.: Kinesio Taping $\left({ }^{\circledR}\right)$ does not alter neuromuscular performance of femoral quadriceps or lower limb function in healthy subjects: randomized, blind, controlled, clinical trial. Man Ther, 2013, 18: 41-45. [Medline] [CrossRef]

8) Fu TC, Wong AM, Pei YC, et al.: Effect of Kinesio taping on muscle strength in athletes-a pilot study. J Sci Med Sport, 2008, 11: 198-201. [Medline] [CrossRef]

9) Wong OM, Cheung RT, Li RC: Isokinetic knee function in healthy subjects with and without Kinesio taping. Phys Ther Sport, 2012, 13: 255-258. [Medline] [CrossRef]

10) Słupik A, Dwornik M, Białoszewski D, et al.: Effect of Kinesio Taping on bioelectrical activity of vastus medialis muscle. Preliminary report. Ortop Traumatol Rehabil, 2007, 9: 644-651. [Medline]

11) Ekiz T, Aslan MD, Özgirgin N: Effects of Kinesio Tape application to quadriceps muscles on isokinetic muscle strength, gait, and functional parameters in patients with stroke. J Rehabil Res Dev, 2015, 52: 323-331. [Medline] [CrossRef]

12) Anandkumar S, Sudarshan S, Nagpal P: Efficacy of kinesio taping on isokinetic quadriceps torque in knee osteoarthritis: a double blinded randomized controlled study. Physiother Theory Pract, 2014, 30: 375-383. [Medline] [CrossRef]

13) Schiffer T, Möllinger A, Sperlich B, et al.: Kinesio taping and jump performance in elite female track and field athletes and jump performance in elite female track and field athletes. J Sport Rehabil, 2015, 24: 47-50. [Medline] [CrossRef]

14) Nunes GS, de Noronha M, Cunha HS, et al.: Effect of kinesio taping on jumping and balance in athletes: a crossover randomized controlled trial. J Strength Cond Res, 2013, 27: 3183-3189. [Medline] [CrossRef]

15) Lumbroso D, Ziv E, Vered E, et al.: The effect of kinesio tape application on hamstring and gastrocnemius muscles in healthy young adults. J Bodyw Mov Ther, 2014, 18: 130-138. [Medline] [CrossRef]

16) Kalron A, Bar-Sela S: A systematic review of the effectiveness of Kinesio Taping — fact or fashion? Eur J Phys Rehabil Med, 2013, 49: 699-709. [Medline]

17) Csapo R, Alegre LM: Effects of Kinesio ${ }^{\circledR}$ taping on skeletal muscle strength—a meta-analysis of current evidence. J Sci Med Sport, 2015, 18: 450-456. [Medline] [CrossRef]

18) Fukui T: Skin taping, skin kinesiology and its clinical appication. Tokyo: Miwa-Shoten, 2015.

19) Fukui T, Otake Y, Kondo T: In which direction does skin move during joint movement? Skin Res Technol, 2016, 22: 181-188. [Medline] [CrossRef]

20) Vithoulka I, Beneka A, Malliou P, et al.: The effects of Kinesio-Taping on quadriceps strength during isokinetic exercise in healthy non athlete women. Isokinet Exerc Sci, 2010, 18: 1-6.

21) Donec V, Varzaityte L, Krisciunas A: The effect of Kinesio Taping on maximal grip force and key pinch force. Pol Ann Med. 2012, 19: 98-105. [CrossRef] 